\title{
Vibrotactile masking and the persistence of tactual features
}

\author{
JAMES C. CRAIG and PAUL M. EVANS \\ Indiana University, Bloomington, Indiana
}

\begin{abstract}
Subjects are more accurate in identifying a vibrotactile pattern when it is presented in isolation than when it is presented in temporal and spatial contiguity with a second vibrotactile pattern. When the temporal separation between the two patterns is relatively long (greater than 200 to $300 \mathrm{msec}$ ), there is more interference when the target pattern is presented second than when it is presented first. It was hypothesized that if features of a vibrotactile pattern persist for relatively long durations, the perception of a subsequent pattern might be interfered with. Two experiments investigated the persistence of tactual features. In both experiments, subjects were presented with two patterns and the temporal separation between the patterns was varied. The subjects were required to identify the second pattern. The results of Experiment 1 showed that at relatively long stimulus onset asynchronies, the subjects often erred and responded with a pattern containing more lines than were contained in the target pattern. Also, the subjects often overestimated the number of lines contained in the target patterns. These findings support the idea that features of vibrotactile patterns persist for relatively long durations and that these features may be integrated with subsequently presented patterns. The results of Experiment 2 indicated that the persisting representation was a veridical copy of the presented pattern and lasted as long as $1,200 \mathrm{msec}$. The results are discussed in terms of a tactile sensory register.
\end{abstract}

A consistent finding in the literature on tactile pattern perception is that it is more difficult to identify a spatial vibrotactile pattern when it is presented in the temporal and spatial proximity of another pattern than when it is presented in isolation (Bliss, Crane, Link, \& Townsend, 1966; Craig, 1985; Evans \& Craig, 1986; Schindler \& Knapp, 1976). The term used to refer to this reduction in the ability to identify one pattern in the presence of another pattern is "masking" (Craig, 1978). Forward masking is said to have occurred when a pattern to be identified is interfered with by a preceding pattern, and backward masking is said to have occurred when a pattern to be identified is interfered with by a subsequently presented pattern. The pattern to be identified is generally termed the target, and the pattern to be ignored is termed the masker.

Using the tactile array of the Optacon (Bliss, Katcher, Rogers, \& Shepard, 1970), Craig (1983) presented subjects with vibrotactile letter-like patterns to their index fingerpads for identification. The patterns were presented in isolation and also in the presence of either a forward or a backward masker. In the masking conditions, the temporal separation between the onsets of the target and masker (stimulus onset asynchrony, or SOA) was varied. As previous studies had shown, there was more backward than forward masking at relatively brief SOAs (100 msec

This research was supported by Grant NS-09783 from the National Institutes of Health. The authors wish to thank Roger Rhodes for his assistance in the conduct of these experiments. Address correspondence to the authors at the Department of Psychology, Indiana University, Bloomington, IN 47405. or less); however, more forward than backward masking was in evidence at SOAs beyond $200 \mathrm{msec}$. A similar result had been reported earlier using air-jet stimuli applied to the fingers (Bliss, Crane, Link, \& Townsend, 1966).

One reason why there might be more forward than backward masking at relatively long SOAs is suggested by a recent study of backward masking (Evans \& Craig, 1986). Using the Optacon array, patterns that varied in the number of lines they contained were presented to subjects' index fingerpads. Following the target presentation, a masking stimulus was presented at one of several different SOAs. At brief SOAs (100 msec or less), subjects often erred and responded with a pattern that contained more lines than were contained in the target pattern. In an estimation task, the effect of the masker was to increase subjects' estimations of the number of lines contained in the target patterns. Both effects decreased as the SOA was increased, and beyond approximately $100 \mathrm{msec}$, subjects' misidentification responses and estimation responses were similar to those made in the absence of the masker. Evans and Craig (1986) attributed these results to the inability of the skin to resolve temporally adjacent patterns. They suggested that information presented to the same site on the skin is integrated over a temporal window of approximately $100 \mathrm{msec}$. As a result, if a backward masker is presented within approximately $100 \mathrm{msec}$ of the onset of a target pattern, the two patterns will be integrated, and subjects will be basing their identifications or estimation decisions on a composite representation containing more lines than were contained in the target pattern. 
An implication of the temporal integration explanation of vibrotactile backward masking is that the presentation of a vibrotactile pattern yields an internal representation that persists for some period of time, at least long enough for the lines of a target pattern to be integrated with those of a subsequently presented masker pattern. In the Evans and Craig (1986) study, each pattern was presented for only $26 \mathrm{msec}$. Thus, the finding of temporal integration at SOAs up to $100 \mathrm{msec}$ implies that a representation of a vibrotactile pattern must persist for at least 70 to $80 \mathrm{msec}$. The possibility exists, however, that the representation of a vibrotactile pattern persists for durations longer than 70 to $80 \mathrm{msec}$. If it does, then one reason why there is more forward than backward masking at relatively long SOAs may be because the persisting representation of the forward masker is integrated with the representation of the target pattern during the integration interval initiated by the target.

There is considerable evidence that the representation of a vibrotactile pattern does persist for relatively long periods following the cessation of stimulation. For example, measurements of the threshold for detecting trains of tactile pulses have shown that, relative to the threshold for a single pulse, the thresholds declined for trains of pulses even when the gap between the pulses was as long as $500 \mathrm{msec}$ (Verrillo, 1965). This result suggests that activity associated with the first pulse persists and is integrated with the activity generated by the subsequent pulse, even when the two stimuli are separated by $500 \mathrm{msec}$. Bliss, Crane, Mansfield, and Townsend (1966) investigated the ability of subjects to report which locations on their fingers had been stimulated. Using a partial-report, whole-report paradigm similar to that developed by Sperling (1960), Bliss et al. found evidence that information about location persisted for as long as $\mathbf{8 0 0 ~ m s e c ~ f o l l o w - ~}$ ing the cessation of stimulation. The results from detection masking studies also point to the persistence of tactile stimuli for up to several hundred milliseconds (Craig, 1978; Gilson, 1969; Sherrick, 1964). The present study investigated the duration and the nature of the persistence of vibrotactile spatial patterns.

Experiment 1 was a replication of the Evans and Craig (1986) study using a forward-masking paradigm. If the presentation of the temporally leading pattern yields a representation that persists for relatively long periods of time, this representation might be integrated with that of the temporally trailing pattern, the target, even when the SOA between the patterns is relatively long. If this is the case, then, at SOAs longer than those observed in Evans and Craig's (1986) backward masking study, we might expect subjects to respond with a pattern containing more lines than are contained in the target pattern and to overestimate the number of lines contained in the target.

\section{EXPERIMENT 1}

Subjects were presented with two vibrotactile patterns in succession. The target patterns are shown in Figure 1. Also shown is the masker that was used. The masker has previously been termed an "energy masker" (Craig, 1978), although the fact that it contains edges and contours may place it somewhere between a "true" energy

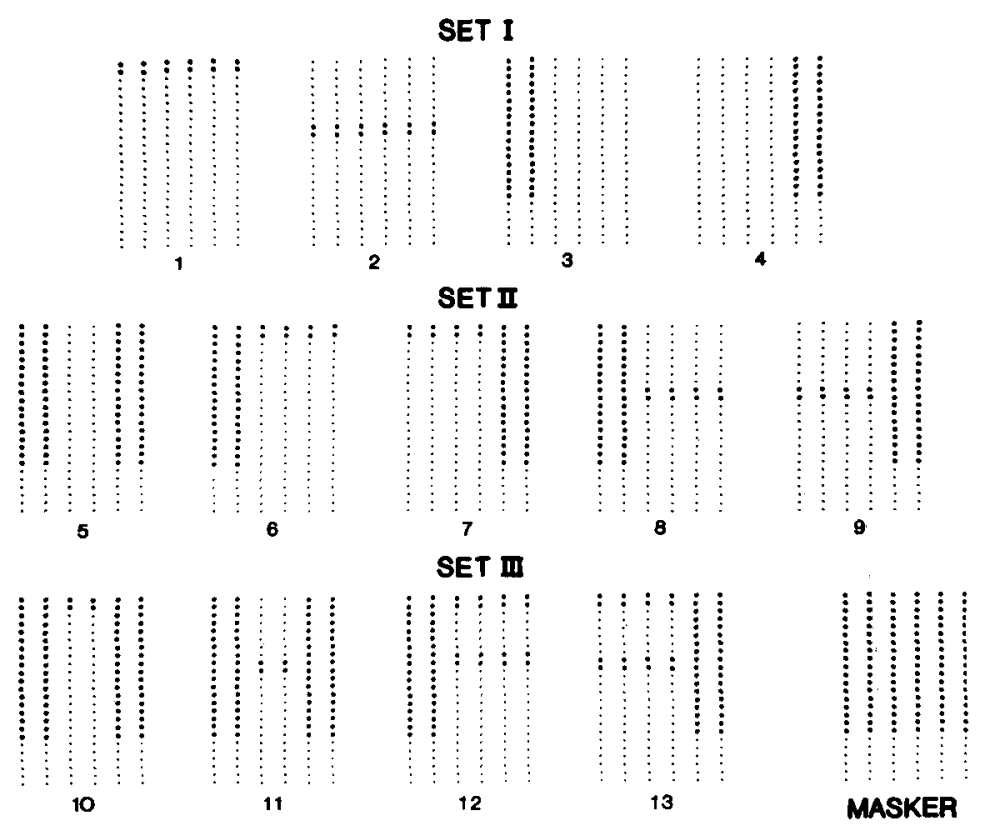

Figure 1. Representations of the patterns used in Experiment 1. (From "Temporal Integration and Vibrotactile Backward Masking" by P. M. Evans and J. C. Craig, 1986, Journal of Experimental Psychology: Human Perception \& Performance, 12, 161. Copyright 1986 by the American Psychological Association. Reprinted by permission.) 
masker and a pattern masker (Craig, 1982), a suggestion supported by the results of Evans and Craig (1986). The target patterns varied in the number of lines they contained. The masker was followed by a target, and the subjects were required to identify the target or to estimate the number of lines contained in the target. Identification and estimation performance in the absence of the masker was also measured.

\section{Method}

Subjects. The subjects were undergraduate students who were paid an hourly rate for their participation. All subjects passed an initial screening test and received experience in vibrotactile pattern perception prior to the beginning of the experiment (Craig, 1982). Four subjects participated in the identification task, and 10 subjects participated in the estimation task. No subject participated in both tasks.

Apparatus. The apparatus consisted of a tactile display controlled by a PDP-11/34 computer. The display was the tactile array of the Optacon, a reading aid for the blind (Bliss et al., 1970). The display consists of 144 pins arranged in a 6-column $\times 24$-row array. Measuring $1.1 \mathrm{~cm}$ in width and $2.7 \mathrm{~cm}$ in height, the display fits against the subject's left index fingerpad. When activated, each pin vibrates at a frequency of about $230 \mathrm{~Hz}$. For additional details of the apparatus, see Craig (1980).

Procedure. The procedural details were identical to those described by Evans and Craig (1986), with the exception that the target was preceded rather than followed by the masker.

In the identification task, the subjects were presented with the masker pattern followed by one of the 13 target patterns and were instructed to ignore the first pattern and to identify the second pattern. Seven SOAs were tested, $-26,-46,-66,-106,-186$, -346 , and -526 msec. Identification performance in the absence of the masker was also measured. Trials were blocked by SOA, feedback was provided on all trials, and an experimental session consisted of eight 40-trial blocks.

In the estimation task, the subjects were presented with the masker pattern followed by one of the target patterns; they were instructed to ignore the first pattern and to estimate the number of lines contained in the second pattern. It was explained to the subjects that each line was made up of two rows or columns of stimulators and that the letter " $E$," for example, contained four lines. Four SOAs were tested, $-26,-46,-106$, and $-346 \mathrm{msec}$. Estimation performance in the absence of the masker was also tested. No feedback was provided. Additional details of both the identification and the estimation task may be found in Evans and Craig (1986). The intensity of the target pattern was set at $32.5 \mathrm{~V}$, the voltage applied to each active pin on the tactile array. The intensity of the masker was also set at $32.5 \mathrm{~V}$.

\section{Results and Discussion}

In the identification task in the absence of the masker, subjects correctly identified the target patterns $82 \%$ of the time. In the presence of the masker, particularly at the briefest SOAs, there was considerable masking. The percent correct identification performance at each SOA was subtracted from the percent correct performance in the absence of the masker to yield a measure of the amount of masking. It is this quantity that is plotted as a function of the temporal separation between the onsets of the target and masker in Figure 2 (forward masking). Each data point is based on an average of 1,600 observations. The standard errors of the means, calculated across blocks of trials and subjects, ranged from $1 \%$ to $4 \%$ in both Experiment 1 and Experiment 2. Also shown in this figure, for comparison purposes, are the results of Evans and Craig's (1986) backward-masking study.

The results plotted in Figure 2 show, as in the Evans and Craig (1986) study, that most masking occurred at the briefest SOAs. At the briefer SOAs there was less forward masking than backward masking, a finding that replicates previous results (Bliss, Crane, Link, \& Townsend, 1966; Craig, 1976, 1978, 1985). In addition, the results show that at longer SOAs there was more forward than backward masking.

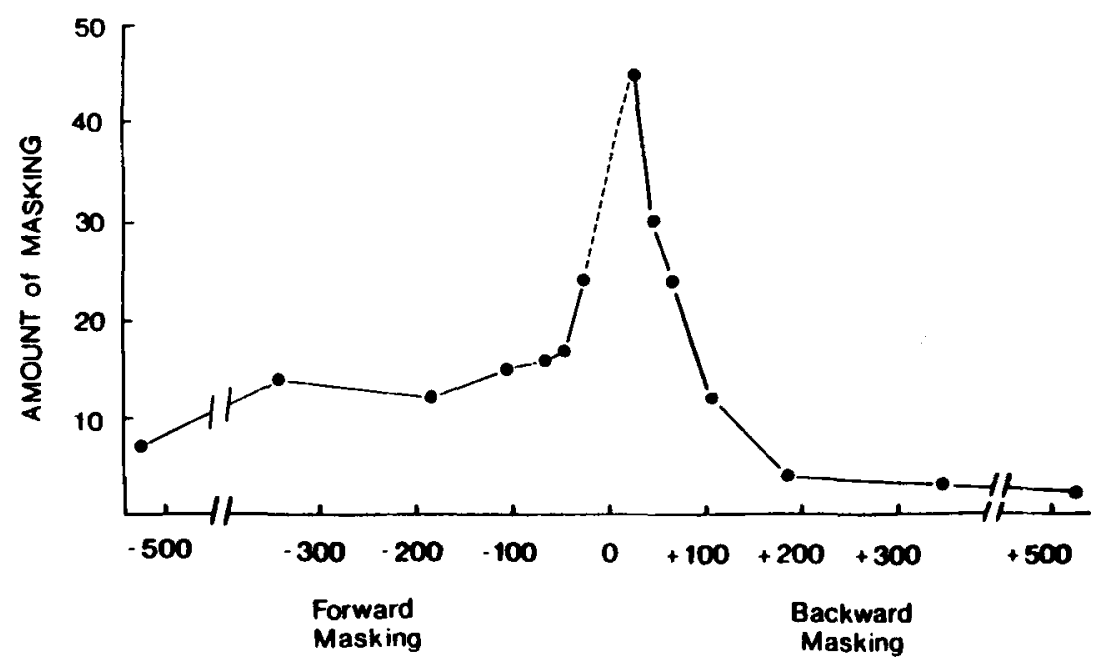

SOA In MSEC

Figure 2. Amount of masking, that is, percent correct obtained in the absence of a masker minus the percent correct obtained in the presence of a masker, plotted as a function of SOA. The backward-masking data are adapted from Evans and Craig (1980). (The dashed line connects the data of the two difrerent groups of subjects.) 
Table 1

Percentage of Responses for Each Response Category

\begin{tabular}{|c|c|c|c|c|c|c|c|c|c|}
\hline \multirow[b]{4}{*}{ SOA } & \multicolumn{9}{|c|}{ Target Pattern } \\
\hline & \multirow{2}{*}{\multicolumn{3}{|c|}{$\frac{\text { One Line }}{\text { Response Category }}$}} & \multicolumn{3}{|c|}{ Two Lines } & \multirow{2}{*}{\multicolumn{3}{|c|}{$\frac{\text { Three Lines }}{\text { Response Category }}$}} \\
\hline & & & & \multicolumn{2}{|c|}{ Response C } & \multirow{2}{*}{$\frac{\text { Category }}{3}$} & & & \\
\hline & 1 & 2 & 3 & 1 & 2 & & 1 & 2 & 3 \\
\hline 26 & $82(81)$ & 13 & 5 & 7 & $60(53)$ & 33 & 2 & 22 & $76(44)$ \\
\hline 46 & $87(86)$ & 11 & 2 & 8 & $67(61)$ & 26 & 1 & 18 & $81(52)$ \\
\hline 66 & $89(88)$ & 9 & 2 & 7 & $72(64)$ & 22 & 2 & 22 & $76(44)$ \\
\hline 106 & $92(91)$ & 8 & 1 & 9 & $75(68)$ & 16 & 2 & 28 & $69(44)$ \\
\hline 186 & $96(95)$ & 3 & 1 & 8 & $76(69)$ & 16 & 2 & 24 & $74(48)$ \\
\hline 346 & $95(94)$ & 4 & 1 & 5 & $80(72)$ & 15 & 2 & 30 & $68(39)$ \\
\hline 526 & $97(96)$ & 3 & 0 & 4 & $84(77)$ & 13 & 1 & 25 & $74(50)$ \\
\hline No Mask & $99(99)$ & 1 & 0 & 4 & $90(86)$ & 7 & 0 & 24 & $76(60)$ \\
\hline
\end{tabular}

Note-SOA = stimulus onset asynchrony (in milliseconds). Numbers in parentheses are the percentages of correct target identifications.

Because the backward-masking results are from an earlier study (Evans \& Craig, 1986) and different groups of subjects were tested in the backward- and forwardmasking conditions (Figure 2), the question might be raised as to whether the greater amount of forward than backward masking observed at the longer SOAs is the result of the temporal position of the masker or the result of differences between the two groups of subjects. Temporal position of the masker appears to be the critical factor for several reasons. First, although two different groups of subjects were tested, the levels of correct performance in the absence of maskers were similar for the two groups, $82 \%$ correct for the subjects in the forwardmasking condition and $85 \%$ correct for the subjects in the backward-masking condition. Second, earlier results obtained from a single group of subjects showed greater forward than backward masking beyond about $400 \mathrm{msec}$ (Craig, 1983). We also tested another group of subjects in a letter-identification task, similar to Condition 1 in Experiment 2, with a pattern masker. Both forward and backward maskers were tested. Once again, at longer SOAs more forward than backward masking was seen and the reverse was true at briefer SOAs. In that study, forward masking exceeded backward masking by $12 \%$ at SOAs of $598 \mathrm{msec}$ and by $12 \%$ at SOAs of $301 \mathrm{msec}$.

When not responding with the target pattern, what types of response errors did subjects make? Specifically, did subjects respond with patterns containing more lines than were presented in the target patterns, suggesting that features of the masker were being integrated with those from the target pattern? To answer this question, the data were

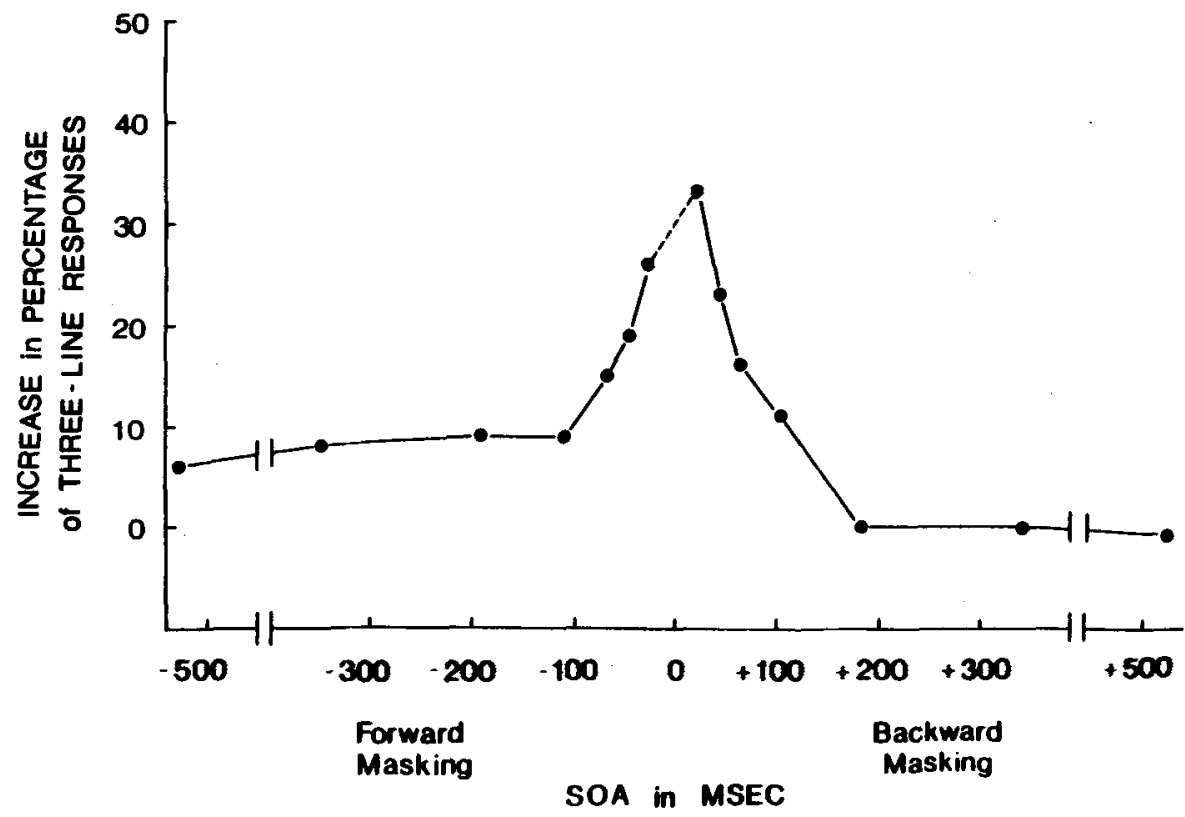

Figure 3. The increment in the percentage of three-line responses to two-line patterns plotted as a function of SOA. The increment was calculated by subtracting the percentage of three-line responses in the absence of a masker from the percentage of three-line responses in the presence of a masker. The backward-masking data are adapted from Evans and Craig (1980). (The dashed line connects the data of the two different groups of subjects.) 
analyzed in terms of the number of lines contained in the target patterns $(1,2$, or 3$)$ and the number of lines given in the subjects' responses $(1,2$, or 3$)$. The results of this analysis are shown in Table 1. For example, when presented with a pattern containing one line preceded by the masker at 26 msec SOA, $82 \%$ of subjects' responses were patterns that contained one line, $13 \%$ were patterns that contained two lines, and 5\% were patterns that contained three lines. The numbers in parentheses are the percentages of correct target identifications.

The data of most interest in Table 1 are those obtained when the target pattern contained two lines. For these targets, the subjects could respond with a pattern indicating that they perceived fewer lines, the same number of lines, or a greater number of lines than were contained in the target pattern. The results show that in the absence of the masker the subjects were more likely, when presented with a target containing two lines, to respond with a threeline target than with a one-line target. However, the magnitude of the effect was relatively small ( $7 \%$ vs. $4 \%$ ), and the majority of the time, the subjects responded with one of the two-line target patterns (90\%).

In the presence of the forward masker, there was a large increase in the percentage of three-line responses to the two-line target patterns. The percentage of one-line responses also increased. However, in the latter case, the increase was relatively small. The increase in the percentage of three-line responses to the two-line target patterns is plotted in Figure 3 as a function of SOA. The increase is measured relative to the percentage of three-line responses made to two-line targets in the absence of a masker. Also plotted are the corresponding results from Evans and Craig's (1986) backward-masking study. In the presence of the backward masker, the increase in the per- centage of three-line responses to the two-line target patterns drops to zero at approximately 100 to $150 \mathrm{msec}$. The results from the present study using a forward masker show that the percentage of three-line responses to the two-line targets remains relatively flat, but greater than zero, out to 526-msec SOA. In other words, at SOAs much longer than those observed in the backward-masking study, subjects were still erring and responding with a pattern that contained more lines than were contained in the target pattern.

The results from the estimation task were analyzed to show how the estimates of the number of lines contained in the target patterns changed as a function of the temporal separation between the onset of the target and the masker. For each of the 13 target patterns at each SOA, the mean number of lines estimated by subjects to be contained in the target pattern was calculated. This mean estimate was subtracted from the mean number of lines estimated by subjects to be contained in the target pattern in the absence of the masker and was multiplied by 100 to obtain the overall percentage overestimation (or underestimation). The resulting function is shown in Figure 4. Also shown are the corresponding data from the Evans and Craig (1986) backward-masking study.

Figure 4 shows that in the presence of either a forward or a backward masker, subjects tended to overestimate the number of lines contained in the target patterns. Moreover, the results are very similar to those shown in Figure 3, in that (1) the amount of overestimation declined with increasing SOA, (2) there was more overestimation at the briefest SOAs with a backward than with a forward masker, and (3) overestimations were observed at relatively longer SOAs with a forward masker than with a backward masker.

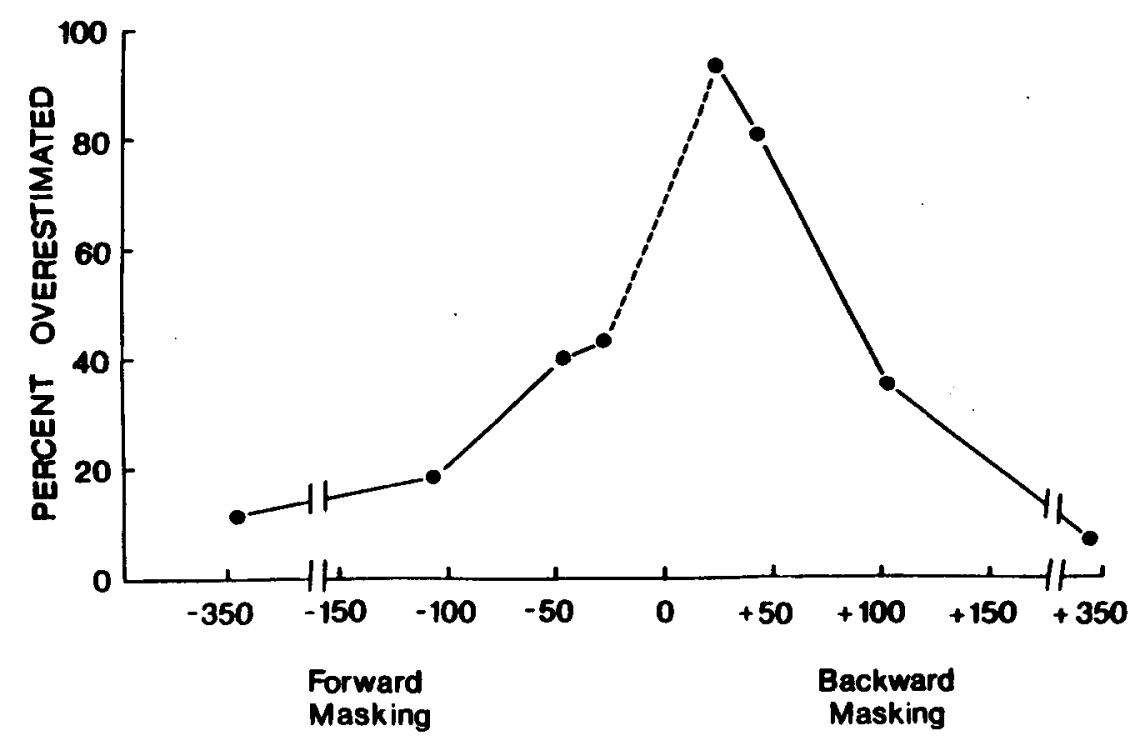

SOA in MSEC

Figure 4. Percentage overestimation of two-line patterns in the presence of a masker as a function of SOA. Overestimation was calculated relative to estimations made in the absence of a masker. The backward-masking data are adapted from Evans and Craig (1986). (The dashed line connects the data of the two different groups of subjects.) 
Together, the results of the identification task and the estimation task support the notion that the presentation of a vibrotactile pattern yields a representation that persists for relatively long periods of time. The elements of the forward masker persist and are integrated with those of the target, causing subjects to respond with a pattern containing more lines than were contained in the target, and to overestimate the number of lines contained in the target pattern. The effects of the masker were similar to those observed in the Evans and Craig (1986) study, with one major difference: the range of SOAs over which the effects were seen was extended.

\section{EXPERIMENT 2}

An energy masker was used in Experiment 1 to permit direct comparisons to be made between the present study and the Evans and Craig (1986) study. In Experiment 2, masker patterns were used that were similar in form to the target patterns (pattern maskers), and a comparison was made between the interfering effect of an energy masker and that of a pattern masker. Both previous results and the results of Experiment 1 of the present study suggest that the energy masker, even though it is generated by turning on all of the pins in the tactile array, contains elements (edges and contours) that are similar to, but not as distinct as, the elements contained in letter and letterlike patterns. Experiment 2 investigated the question of whether the presence of distinct elements in the masker patterns would extend the time interval over which temporal integration was observed.

The use of pattern maskers in Experiment 2 enabled a second question to be addressed. If the representation of a vibrotactile pattern persists for relatively long dura- tions, is information about the spatial locations of the elements contained in the representation preserved? If it were, then we would expect little or no interference when the masker pattern was identical to the target pattern, since the integration of two identical patterns will yield a composite form that is identical to the pattern to be identified.

To answer these two questions, forward masking at long SOAs was examined in three conditions. The first condition compared energy and pattern maskers. The second and third conditions used letters as both targets and maskers. On some proportion of the trials in the latter two conditions, the targets and maskers were identical.

\section{Method}

Subjects. Groups of subjects were selected from the pool of subjects in the laboratory according to the subjects' availability. The number of subjects for each set of measurements is noted in the procedure section.

Procedure. The general procedures were similar to those described for Experiment 1. The identifiability of target patterns was determined in the presence and absence of masking patterns at various SOAs.

Three conditions were used. In Condition 1, the effectiveness of an energy masker in interfering in letter recognition was compared with the effectiveness of a pattern masker. The maskers were used as forward maskers at SOAs of $-1,200,-600$, and $-300 \mathrm{msec}$. The pattern maskers were generated as they had been in previous studies (Craig, 1982) by selecting randomly, on a trial-by-trial basis, from among 26 patterns. Preliminary work had shown only a slight interference in pattern recognition by pattern maskers at long SOAs. To enhance this effect, we increased the intensity of the maskers by increasing the duration of the maskers from 26 to $100 \mathrm{msec}$ and by increasing the voltage supplied to the individual pins. For the pattern masker, the voltage was increased from 32.5 to $50 \mathrm{~V}$. The intensity of the energy masker was adjusted to be equal in perceived intensity to the pattern masker. The voltage was set at $36 \mathrm{~V}$ for the energy masker. Five subjects were tested.

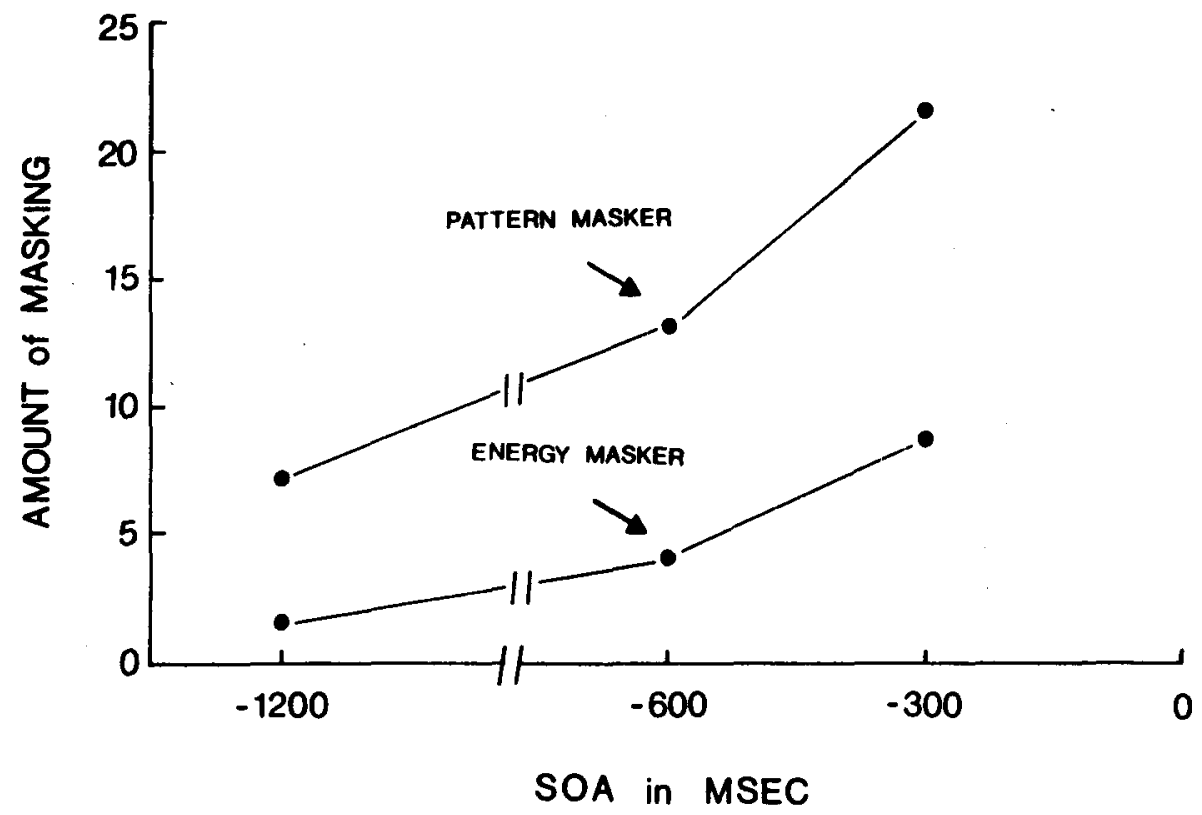

Figure 5. Amount of masking, as defined for Figure 2, in the presence of a forward masker as a function of SOA. Both pattern and energy maskers were tested. 
In Condition 2, an energy masker and pattern maskers, in this case, letters of the alphabet, were used. Letters were also used as target patterns, so that on some proportion of the trials in which letters were used as maskers, the target and masker would be identical. By chance, a match would occur on about $4 \%$ of the trials, 1 in 26. To have sufficient data to analyze the same pairs, the percentage of trials in which a match occurred was increased to $20 \%$. The subjects were instructed, as they were in all the masking tasks, to ignore the masker; they were not told that on some proportion of the trials the maskers would be identical to the targets. In Condition 2, as in Condition 1, a forward-masking paradigm was used with SOAs of $-1,200,-600$, and -300 msec. A no-mask condition was included. The duration, $100 \mathrm{msec}$, and the intensity levels, $50 \mathrm{~V}$ for the letter maskers and $36 \mathrm{~V}$ for the energy masker, were the same as those used in Condition 1. Six subjects were tested.

In Condition 3, five letters were selected as targets and the same five letters were used as maskers. The letters selected, BEMRW, are among the more difficult letters to identify tactually (Craig, 1979). When used as maskers, the letters were set at a greater intensity, $50 \mathrm{~V}$ for $100 \mathrm{msec}$, than when they were used as targets. On any trial, the selection of a particular target and masker pair was determined randomly. Hence, the percentage of trials on which the two were identical was $20 \%$. Three subjects were tested.

\section{Results and Discussion}

The results from Condition 1 are shown in Figure 5. Each point represents a total of 1,600 trials from 5 subjects. The results are presented in terms of the amount of masking, that is, the difference between percent correct identification in the no-mask condition, which was $66 \%$, and the percent correct identification in each of the masker conditions.

Condition 1 addressed the persistence of the representation of a vibrotactile pattern at long SOAs. It appears that a vibrotactile representation does persist for temporal intervals in excess of the 500-msec intervals tested in Experiment 1 . Evidence for this assertion is that there is both a significant amount of masking with the pattern masker at 300-, 600-, and 1,200-msec SOA $(p<.01)$ and a significantly greater amount of masking with the pattern masker than with the energy masker at $300(p<.001)$, at $600(p<.01)$, and at $1,200 \mathrm{msec}(p<.05$, MannWhitney $U$ test). The Mann-Whitney $U$ test was performed by ranking the data according to the level of performance on all blocks of trials by all subjects for a particular condition. All data analysis in Experiment 2 was performed in a similar manner. The energy masker did not produce significant amounts of masking at either 600 or 1,200-msec SOAs. In short, two maskers differing in featural content but equal in perceived intensity have a differential effect on the identification of a spatial target, and this differential effect continues out to temporal separations as long as $1,200 \mathrm{msec}$.

The results from Condition 2 are shown in Figure 6. In the absence of a masker, performance was $72 \%$ correct. For the energy mask conditions, each point represents the results from 6 subjects, 3,120 trials. The total number of trials for the letter masker condition was 3,120 overall. Because the masker was different from the target on $80 \%$ of the trials, each data point here represents 2,496 trials $[(3120)(.80)]$. For the conditions in which the target and masker were identical, each data point represents 624 trials $[(3120)(.20)]$. Letter maskers different from the target produced significantly more masking than energy maskers at all SOAs $(p<.01)$, and significantly more interference than identical letter maskers at 300- and 600 -msec SOAs $(p<.001)$.

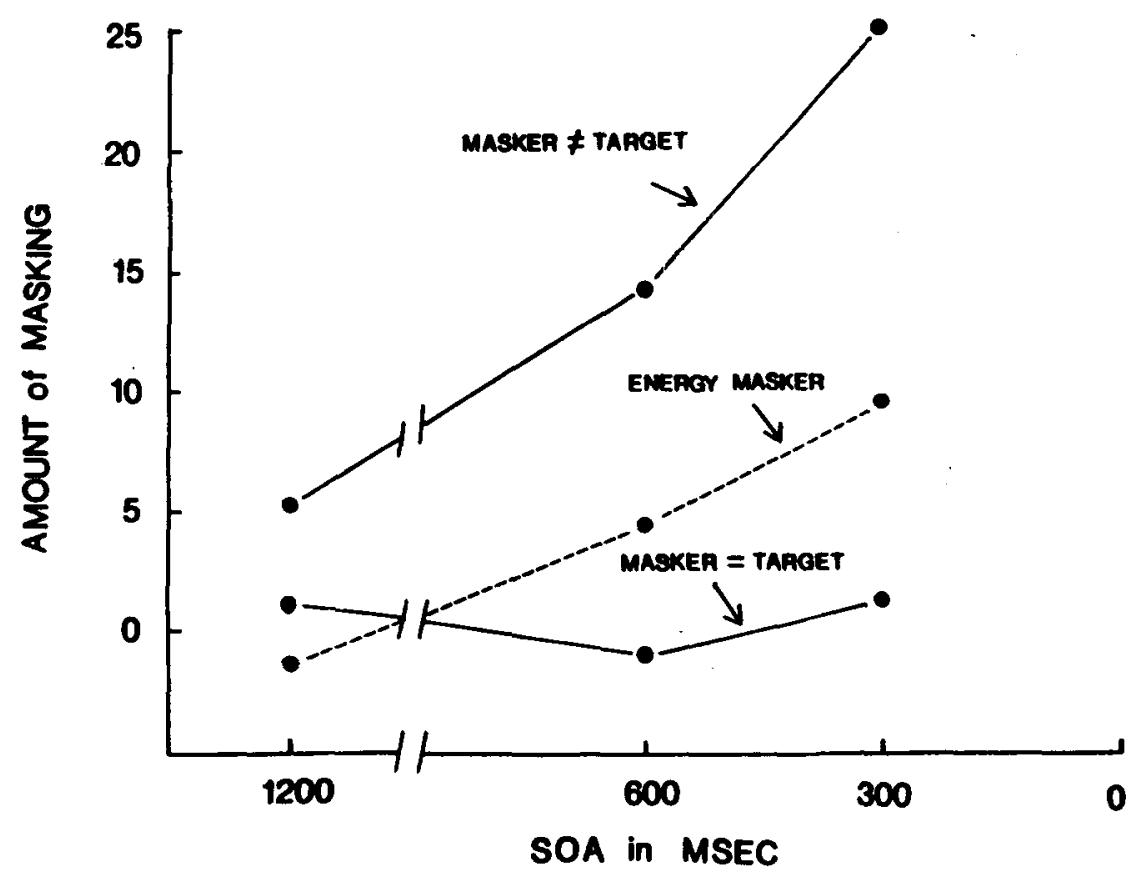

Figure 6. Amount of masking, as defined for Figure 2, in the presence of a forward masker as a function of SOA. Three types of maskers were tested: pattern maskers identical to the target, pattern maskers not identical to the target, and energy maskers. 


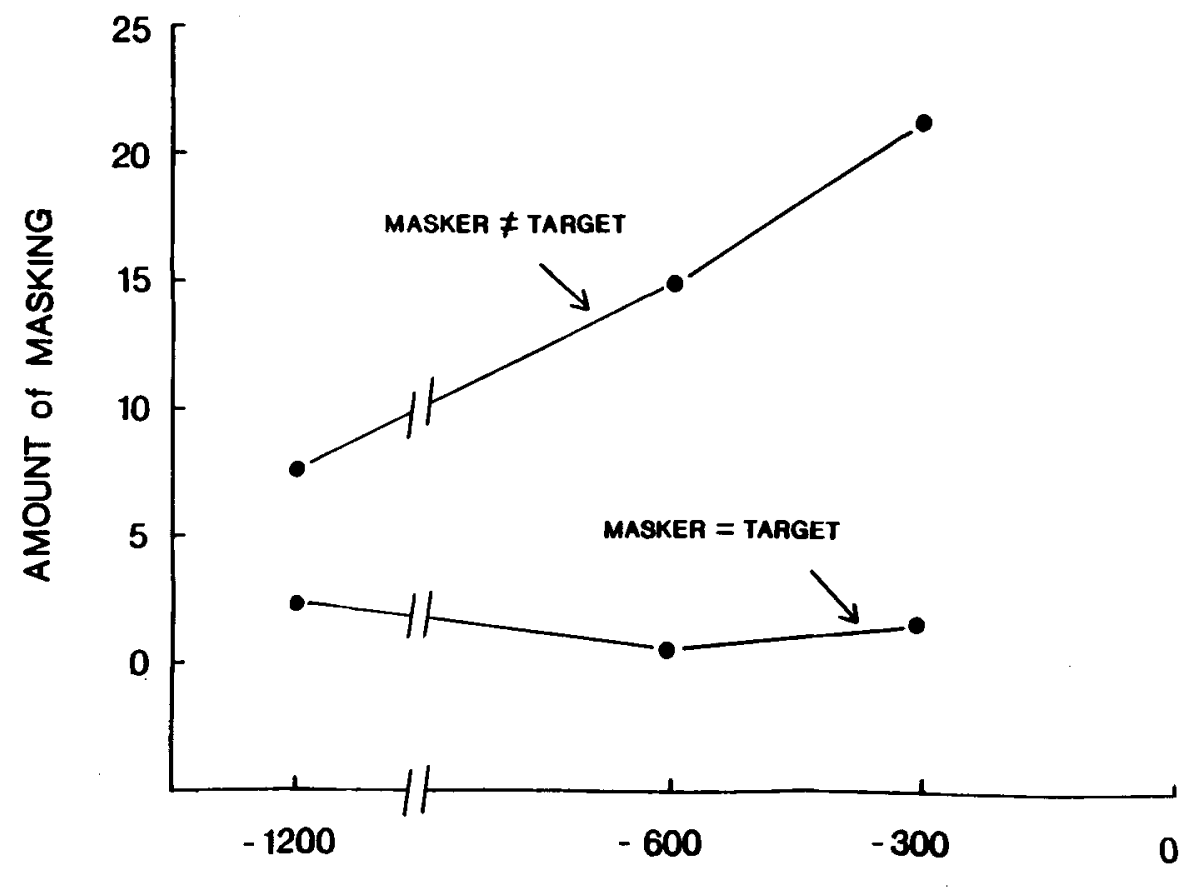

\section{SOA in MSEC}

Figure 7. Similar to Figure 6. Two types of pattern maskers were tested: maskers identical to the target and maskers not identical to the target.

These results extend the findings of Experiment 1 and Condition 1 of Experiment 2 by suggesting that not only does the representation of a vibrotactile pattern persist for relatively long times, but also information about the spatial locations of lines contained in the representation is preserved. This conclusion is based on the fact that although patterned stimuli (different letters) produce considerable interference at $-600 \mathrm{msec}$, identical letters do not. It is as though a relatively faithful copy of the masker is preserved for at least $600 \mathrm{msec}$ and either matches (no masking) or fails to match (masking) the target letter.

In Condition 3, the number of possible targets and maskers was reduced from 26 to 5 letters. The results from 3 subjects are shown in Figure 7. Performance in the absence of a masker was $78 \%$ correct based on a total of 2,640 trials. In the masking conditions, $80 \%$ of the trials, 2,112 , were ones in which the target and masker were different, and $20 \%$ of the trials, 528 , were ones in which the two were identical. There was significantly more masking at all SOAs when the maskers differed from the targets than when the maskers and targets were identical $(p<.001$ at 300 and $600 \mathrm{msec}, p<.05$ at $1,200 \mathrm{msec}$ ). It might be argued that in Condition 2, described above, the fact that the masker was identical to the target on a proportion of trials greater than chance permitted the subjects to gain some information about the target from the masker. The fact that in neither Condition 2 nor Condition 3 did performance when the masker was identical to the target improve significantly over performance in the absence of a masker suggests that subjects make little use of information from the masker.
However, an analysis of the trials in which the masker was different from the target showed subjects' responding, incorrectly, with the masker at greater than chance levels. It is likely that on some trials the clearest representation available to the subject is the masker; but, this tendency to respond with the masker had only a small effect on the amount of masking observed. For example, at $-600 \mathrm{msec}$ in Condition 3, had subjects responded with the masker at chance levels, the amount of masking would have declined by approximately $3 \%$.

\section{GENERAL DISCUSSION}

Perhaps the best explanation of the results of the present study is that the presentation of a vibrotactile pattern yields an internal representation that persists in a fashion similar to the persistence of a visual pattern in iconic memory. If such a vibrotactile sensory register does exist, what might some of its characteristics be? First, the fact that interference is greatest at SOAs below $100 \mathrm{msec}$ and decreases precipitously thereafter suggests that information contained within the tactile sensory store decays rapidly at first and then at a slower rate. Second, the fact that interference is observed at SOAs up to $1,200 \mathrm{msec}$ suggests that the duration of the tactile sensory store is at least $1,200 \mathrm{msec}$ and perhaps even longer. Third, the finding, in Experiment 2, that little interference was observed when the masker pattern was identical to the target pattern suggests that information contained within the tactile sensory store is preserved in a relatively intact form, that is, veridical with respect to the spatial locations of 
the features of patterns. Fourth, judging by the relatively small amounts of masking observed at the longer SOAs, the representation of the tactile patterns is relatively weak at the longer SOAs.

In arriving at the conclusion that the presentation of a vibrotactile stimulus yields an internal representation that persists following the cessation of stimulation, two alternative explanations of the results of the present study were considered: first, that the skin itself retains an impression of the pattern of stimulation, or second, that the array of tactile stimulators continues to provide a mechanical stimulus to the skin after the electrical signal to the array has been turned off. With regard to the first explanation, it is true that if a sharp object is pressed into the fingertip, the skin may take several seconds before returning to its original state. Returning to its prestimulation state might produce neural signals similar to those produced during actual stimulation. However, microscopic examination of the skin during contact with the stimulators and following the offset of stimulation did not show any significant, long-lasting compression of the skin. In addition, if the skin were subjected to significant compression and then gradually returned to its prestimulated state, the gradual movement of the skin over a period of 1,200 msec would be of a very low frequency to which the skin is insensitive. It is unlikely that the results of the present study are due to the skin's retaining an impression of the presented stimulus.

With regard to the second explanation, perhaps a physical representation of the presented stimulus outlasts the offset of the electrical signal to the tactile display. To examine the possibility that pins continue to vibrate after the offset of the electrical signal, an accelerometer was placed against the tactile display. The vibratory response lasted only a few milliseconds after the offset of the electrical signal. It is also possible that the pins of the tactile array might not return to their prior, unstimulated position at the offset of the electrical signal but continue to protrude into the skin. To check for a slowly decaying dc displacement that might remain after the signal offset, we examined the mechanical motion of the display with a binocular microscope under both stroboscopic and regular illumination. We noted that the stimulators returned to their resting dc positions at the offset of the electrical signal. In short, there was no indication that a physical representation of the stimulus outlasted the offset of the electrical signal to the display.

To conclude, the findings of the present study suggest that the reason there is more forward than backward masking at relatively long SOAs (beyond 200 to $300 \mathrm{msec}$ ) is that the representation of a vibrotactile pattern persists in a tactile sensory store for a relatively long duration and that, as a result, the elements of a forward masker pat- tern interfere with those of a subsequently presented target pattern. Experiment 1 showed that at SOAs out to $500 \mathrm{msec}$ the nature of the interference was that the elements of the masker were integrated with those of the target. The results of Experiment 2 suggest that the representation of a vibrotactile pattern is veridical with respect to the spatial locations of the elements contained within the pattern. Because of the nature of the patterns used in Experiment 2, we cannot say with certainty that the masking observed at SOAs longer than 500 msec was the result of integration of features from the masker with those from the target pattern. It may be that factors other than integration produce interference with forward maskers at SOAs longer than 500 msec.

\section{REFERENCES}

Buiss, J. C., Crane, H. D., Link, S. W., \& Townsend, J. T. (1966). Tactile perception of sequentially presented spatial patterns. Perception \& Psychophysics, 1, 125-130.

Buss, J. C., Crane, H. D., Mansfield, P. K., \& Townsend, J. T. (1966). Information available in brief tactile presentations. Perception \& Psychophysics, 1, 273-283.

Bliss, J. C., Katcher, M. H., Rogers, C. H., \& Shepard, R. P. (1970). Optical-to-tactile image conversion for the blind. IEEE Transactions on Man-Machine Systems, MMS-11, 58-64.

Craig, J. C. (1976). Vibrotactile letter recognition: The effects of a masking stimulus. Perception \& Psychophysics, 20, 317-326.

Craig, J. C. (1978). Tactile pattern recognition and masking. In G. Gordon (Ed.), Active touch: The mechanism of recognition of objects by manipulation: A multi-disciplinary approach (pp. 229-242). Oxford, England: Pergamon Press.

Craig, J. C. (1979). A confusion matrix for tactually presented letters. Perception \& Psychophysics, 26, 409-411.

CRAIG, J. C. (1980). Modes of vibrotactile pattern perception. Journal of Experimental Psychology: Human Perception \& Performance, 6 , 151-166.

Craig, J. C. (1982), Vibrotactile masking: A comparison of energy and pattern maskers. Perception \& Psychophysics, 31, 523-529.

Craig, J. C. (1983). The role of onset in the perception of sequentially presented vibrotactile patterns. Perception \& Psychophysics, 34, $421-432$.

CRAIG, J. C. (1985). Tactile pattern perception and its perturbations. Journal of the Acoustical Society of America, 77, 238-246.

Evans, P. M., \& CRAIG, J. C. (1986). Temporal integration and vibrotactile backward masking. Journal of Experimental Psychology: Human Perception \& Performance, 12, 160-168.

Gruson, R. D. (1969). Vibrotactile masking: Some spatial and temporal aspects. Perception \& Psychophysics, 5, 176-180.

SCHINDLER, U., \& KNAPP, A. (1976). Ursachen der gegenseitigen Verdeckung von taktil dargebotenen Buchstaben: Unterbrechung, Summation, oder Verzogerung. Psychological Research, 38, 303-318.

SHERRICK, C. E. (1964). Effects of double simultaneous stimulation of the skin. American Journal of Psychology, 77, 42-53.

SPERLING, G. (1960). The information available in brief visual presentations. Psychological Monographs, 74, 1-29.

VERRILLO, R. T. (1965). Temporal summation in vibrotactile sensitivity. Journal of the Acoustical Society of America, 37, 843-846.

(Manuscript received December 2, 1986; revision accepted for publication March 27,1987 .) 